\title{
Pricing and hedging contingent claims with liquidity costs and market impact
}

\author{
Frederic Abergel and Grégoire Loeper \\ Chair of Quantitative Finance \\ Laboratory of Mathematics Applied to Systems, \\ École Centrale Paris, 92290 Châtenay Malabry, France
}

April 13, 2013

\begin{abstract}
We study the influence of taking liquidity costs and market impact into account when hedging a contingent claim, first in the discrete time setting, then in continuous time. In the latter case and in a complete market, we derive a fully non-linear pricing partial differential equation, and characterizes its parabolic nature according to the value of a numerical parameter naturally interpreted as a relaxation coefficient for market impact. We then investigate the more challenging case of stochastic volatility models, and prove the parabolicity of the pricing equation in a particular case.
\end{abstract}

\section{Introduction}

There is a long history of studying the effect of transaction costs and liquidity costs in the context of derivative pricing and hedging. Transaction costs due to the presence of a Bid-Ask spread are well understood in discrete time, see [8]. In continuous time, they lead to quasi-variational inequalities, see e.g. [17], and to imperfect claim replication due to the infinite cost of hedging continuously over time. In this work, the emphasis is put rather on liquidity costs, that is, the extra price one has to pay over the theoretical price of a tradable asset, due to the finiteness of available liquidity at the best possible price. A reference work for the modelling and mathematical study of liquidity in the context of a dynamic hedging strategy is [3], see also [14, and our results can be seen as partially building on the same approach.

It is however unfortunate that a major drawback occurs when adding liquidity costs: as can easily be seen in [3] [12] [14, the pricing and hedging equation are not unconditionally parabolic anymore and, therefore, only a local existence and uniqueness of smooth solutions may be available. Note that this drawback can easily be inferred from the very early heuristics in [9]: the formula suggested by Leland makes perfectly good sense for small perturbation of the initial volatility, but is meaningless when the modified volatility becomes negative. An answer to this problem is proposed in [4], where the authors introduce super-replicating strategies and show that the minimal cost of a super-replicating strategy solves a well-posed parabolic equation. Still, a partial conclusion is that incorporating liquidity cost leads to ill-posed pricing equation for large option positions, a situation which cannot be considered satisfactory and hints at the fact that some ingredient may be missing in the physical modelling of the market. It turns out that this missing ingredient is precisely the market impact of the delta-hedger, as will become clear from our results. This fact is already observed by the second author in [11, where a well posed, fully non-linear parabolic equation is obtained using a simple market impact model.

Motivated by the need for quantitative approaches to algorithmic trading, the study of market impact in order-driven markets has become a very active research subject in the past decade. In a very elementary way, there always is an instantaneous market impact - termed virtual impact in [16] - whenever a transaction 
takes place, in the sense that the best available price immediately following a transaction may be modified if the size of the transaction is larger than the quantity available at the best limit in the order book. As many empirical works show, see e.g. [2] [16], a relaxation phenomenon then takes place: after a trade, the instantaneous impact decreases to a smaller value, the permanent impact. This phenomenon is named resilience in [16], it can be interpreted as a rapid, negatively correlated response of the market to large price changes due to liquidity effects. In the context of derivative hedging, it is clear that there are realistic situations - e.g., a large option on an illiquid stock - where the market impact of an option hedging strategy is significant. This situation has already been addressed by several authors, see in particular [15 [6] [5] [13], where various hypothesis on the dynamics, the market impact and the hedging strategy are proposed and studied. One may also refer to [7 [10] 14 for more recent related works. It is however noteworthy that in these references, liquidity costs and market impact are not considered jointly, whereas in fact, the latter is a rather direct consequence of the former. As we shall demonstrate, the level of permanent impact plays a fundamental role in the well-posedness of the pricing and hedging equation, a fact that was overlooked in previous works on liquidity costs and impact. Also, from a practical point of view, it seems relevant to us to relate the well-posedness of the modified Black-Scholes equation to a parameter that can be measured empirically using high frequency data.

This paper aims at contributing to the field by laying the grounds for a reasonable model of liquidity costs and market impact for derivative hedging. We start in a discrete time setting, where notions are best introduced and properly defined, and then move on to the continuous time case. Liquidity costs are modelled by a simple, stationary order book, characterized by its shape around the best price, and the permanent market impact is measured by a numerical parameter $\gamma, 0 \leqslant \gamma \leqslant 1: \gamma=0$ means no permanent impact, so the order book goes back to its previous state after the transaction is performed, whereas $\gamma=1$ means no relaxation, the liquidity consumed by the transaction is not replaced. This simplified representation of market impact rests on the hypothesis that the characteristic time of the derivative hedger may be different from the relaxation time of the order book, a realistic hypothesis since delta-hedge generally occurs at a lower frequency than does liquidity providing.

What we consider as our main result is Theorem 4.1. which states that, in the complete market case, the range of parameter for which the pricing equation is unconditionally parabolic is $\frac{2}{3} \leqslant \gamma \leqslant 1$. This result, which we find quite nice in that it is explicit in terms of the parameter $\gamma$, obviously explains the ill-posedness

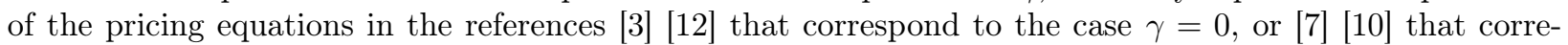
spond to the case to the case $\gamma=\frac{1}{2}$ within our formulation. In particular, Theorem 4.1 implies that when re-hedging occurs at the same frequency as that at which liquidity is provided to the order book - that is, when $\gamma=1$ - the pricing equation is well-posed. This result was already obtained by the second author in [11].

The paper is organized as follows: after recalling some classical notations and concepts, Section 1 presents the order book model that will be used to describe liquidity costs. Then, in Section 2, we write down the model for the observed price dynamics and study the associated risk-minimizing strategy taking into account liquidity costs and market impact. Section 3 is devoted to the continuous time version of these results. The pricing and hedging equations are then worked out and characterized in the case of a complete market, in the single asset case in Section 4 and in the multi-asset case in Section 5 . Finally, Section 6 touches upon the case of stochastic volatility models, for which partial results are presented.

\section{Basic notations and definitions}

To ease notations, we will assume throughout the paper that the risk-free interest rate is always 0 , and that the assets pay no dividend.

\section{Discrete time setting}

The tradable asset price is modelled by a stochastic process $S_{k},(k=0, \cdots, T)$ on a probability space $(\Omega, \mathcal{F}, P) . \mathcal{F}_{k}$ denotes the $\sigma$-field of events observable up to and including time $k . S_{k}$ is assumed to be 
adapted and square-integrable.

A contingent claim is a square-integrable random variable $H \in L^{2}(P)$ of the following form $H=\delta^{H} S_{T}+\beta^{H}$ with $\delta^{H}$ and $\beta^{H}, \mathcal{F}_{T}$-measurable random variables.

A trading strategy $\Phi$ is given by two stochastic processes $\delta_{k},(k=0, \cdots, T)$ and $\beta_{k},(k=0, \cdots, T) . \delta_{k}$ (resp. $\left.\beta_{k}\right)$ is the amount of stock (resp. cash) held during period $k,\left(=\left[t_{k}, t_{k+1}\right)\right)$ and is fixed at the beginning of that period, i.e. we assume that $\delta_{k}$ (resp. $\left.\beta_{k}\right)$ is $\mathcal{F}_{k}-$ measurable $(k=0, \cdots, T)$. Moreover, $\delta$ and $\beta$ are in $L^{2}(P)$.

The theoretical value of the portfolio at time $k$ is given by

$$
V_{k}=\delta_{k} S_{k}+\beta_{k},(k=1, \cdots, T) .
$$

A strategy is $H$-admissible iff each $V_{k}$ is square-integrable and $V_{T}=H$.

\section{Continuous time setting}

In the continuous case, $(\Omega, \mathcal{F}, P)$ is a probability space with a filtration $\left(\mathcal{F}_{t}\right)_{0 \leq t \leq T}$ satisfying the usual conditions of right-continuity and completeness. $T \in \mathbb{R}^{*+}$ denotes a fixed and finite time horizon. Moreover, $\mathcal{F}_{0}$ is trivial and $\mathcal{F}_{T}=\mathcal{F}$.

The risky asset $S=\left(S_{t}\right)_{0 \leq t \leq T}$ is a strictly positive, continuous $\mathcal{F}_{t}$-semimartingale, and a trading strategy $\Phi$ is a pair of càdlàg and adapted processes $\delta=\left(\delta_{t}\right)_{0 \leq t \leq T}, \beta=\left(\beta_{t}\right)_{0<t \leq T}$, while a contingent claim is described by a random variable $H \in L^{2}(P)$, with $H=\delta^{H} S_{T}+\beta^{H}, \delta^{H}$ and $\beta^{\bar{H}}$ being $\mathcal{F}_{T}$-measurable random variables. $H$-admissible strategies are defined as follows:

Definition 1.0.1 A trading strategy will be called $H$-admissible iff

$$
\left\{\begin{array}{l}
\delta_{T}=\delta^{H} P-\text { a.s. } \\
\beta_{T}=\beta^{H} P-\text { a.s. } \\
\delta \text { has finite and integrable quadratic variation } \\
\beta \text { has finite and integrable quadratic variation } \\
\delta \text { and } \beta \text { have finite and integrable quadratic covariation. }
\end{array}\right.
$$

\section{Order book, transaction cost and impact}

A constant, symmetric order-book profile is considered around the price $\hat{S}_{t}$ of the asset $S$ at a given time $t$ before the option position is delta-hedged - think of $\hat{S}_{t}$ as a theoretical price in the absence of the option hedger. The relative density $\mu(x) \geqslant 0$ of the order book is the derivative of the function $M(x) \equiv \int_{0}^{x} \mu(t) d t \equiv$ number of shares one can buy (resp. sell) between the prices $\hat{S}_{t}$ and $\hat{S}_{t}(1+x)$ for positive (resp. negative) $x$. The instantaneous - virtual in the terminology of [16] - market impact of a transaction of size $\epsilon$ is then

$$
I_{\text {virtual }}(\epsilon)=\hat{S}_{t} M^{-1}(\epsilon),
$$

it is precisely the difference between the price before and immediately after the transaction is completed. The level of permanent impact is then measured via a parameter $\gamma$ :

$$
I_{\text {permanent }}(\epsilon)=\gamma \hat{S}_{t} M^{-1}(\epsilon) .
$$

It seems reasonable to assume that $0 \leqslant \gamma \leqslant 1$, but we do not impose a priori this constraint. The actual cost of the same transaction is

$$
C(\epsilon)=\hat{S}_{t} \int_{0}^{M^{-1}(\epsilon)}(1+x) \mu(x) d x \equiv \hat{S}_{t} \int_{0}^{\epsilon}\left(1+M^{-1}(y)\right) d y .
$$

We denote by $\kappa$ the function $M^{-1}$. 


\section{Cost process with market impact in discrete time}

In this section, we focus on the discrete time case.

\subsection{The observed price dynamics}

The model for the dynamics of the observed price - that is, the price $S_{k}$ that the market can see at every time $t_{k}$ after the re-hedging is complete - is now presented.

A natural modelling assumption is that the price moves according to the following sequence of events:

- First, it changes under the action of the "market" according to some (positive) stochastic dynamics for the theoretical price increment $\Delta \hat{S}_{k}$

$$
\hat{S}_{k} \equiv S_{k-1}+\Delta \hat{S}_{k} \equiv S_{k-1}\left(1+\Delta M_{k}+\Delta A_{k}\right),
$$

where $\Delta M_{k}$ (resp. $\Delta A_{k}$ ) is the increment of an $\mathcal{F}$-martingale (resp. an $\mathcal{F}$-predictable process).

- Then, the hedger applies some extra pressure by re-hedging her position, being thereby subject to liquidity costs and market impact as introduced in Section 1. As a consequence, the dynamics of the observed price is

$$
S_{k}=S_{k-1}\left(1+\Delta M_{k}+\Delta A_{k}\right)\left(1+\gamma \kappa\left(\delta_{k}-\delta_{k-1}\right)\right) .
$$

\subsection{Incremental cost of the hedging strategy}

Following the approach developed in [12], the incremental cost $\Delta C_{k}$ of re-hedging at time $t_{k}$ is now studied: the strategy consists in buying $\delta_{k}-\delta_{k-1}$ shares of the asset, and rebalancing the cash account from $\beta_{k-1}$ to $\beta_{k}$. With the notations just introduced in Section 2.1 there holds

$$
\Delta C_{k}=\hat{S}_{k} \int_{0}^{\delta_{k}-\delta_{k-1}}(1+\kappa(u)) d u+\left(\beta_{k}-\beta_{k-1}\right)
$$

Using the value process

$$
V_{k}=\beta_{k}+\delta_{k} S_{k} \equiv \beta_{k}+\delta_{k} \hat{S}_{k}\left(1+\gamma \kappa\left(\delta_{k}-\delta_{k-1}\right)\right),
$$

one can then rewrite the incremental cost between $t_{k-1}$ and $t_{k}$ as

$$
\Delta C_{k}=\left(V_{k}-V_{k-1}\right)-\left(\delta_{k} S_{k}-\delta_{k-1} S_{k-1}\right)+\hat{S}_{k} \int_{0}^{\delta_{k}-\delta_{k-1}}(1+\kappa(u)) d u .
$$

Straightforward computations lead to

$$
\Delta C_{k}=\left(V_{k}-V_{k-1}\right)-\delta_{k-1}\left(S_{k}-S_{k-1}\right)+S_{k}\left(\frac{\int_{0}^{\delta_{k}-\delta_{k-1}} 1+\kappa(u) d u}{1+\gamma \kappa\left(\delta_{k}-\delta_{k-1}\right)}-\left(\delta_{k}-\delta_{k-1}\right)\right),
$$

or equivalently

$$
\Delta C_{k}=\left(V_{k}-V_{k-1}\right)-\delta_{k-1}\left(S_{k}-S_{k-1}\right)+S_{k}\left(\frac{\int_{0}^{\delta_{k}-\delta_{k-1}} \kappa(u) d u-\gamma\left(\delta_{k}-\delta_{k-1}\right) \kappa\left(\delta_{k}-\delta_{k-1}\right)}{1+\gamma \kappa\left(\delta_{k}-\delta_{k-1}\right)}\right) .
$$

To ease the notations, let us define, for $x \in \mathbf{R}$,

$$
\mathbf{g}(x) \equiv \frac{\int_{0}^{x} \kappa(u) d u-\gamma x \kappa(x)}{1+\gamma \kappa(x)} .
$$


The function $\mathbf{g}$ is smooth and satisfies

$$
\mathbf{g}(0)=\mathbf{g}^{\prime}(0)=0, \mathbf{g}^{\prime \prime}(0)=(1-2 \gamma) \kappa^{\prime}(0),
$$

thanks to the obvious property $\kappa(0)=0$ for the instantaneous price impact function.

We summarize our results in the

Proposition 2.1 The incremental cost of implementing a hedging strategy at time $t_{k}$ has the following expression

$$
\Delta C_{k}=\left(V_{k}-V_{k-1}\right)-\delta_{k-1}\left(S_{k}-S_{k-1}\right)+S_{k} \mathbf{g}\left(\delta_{k}-\delta_{k-1}\right),
$$

where the function $\mathbf{g}$ is given in 2.8 .

\subsection{Optimality conditions for quadratic local-risk minimization}

Proposition (2.1) is the starting point for the characterization of a local-risk minimizing strategy. Upon using a quadratic criterion, and under some assumptions ensuring the convexity of the quadratic risk, see e.g. [12], one easily derives the two (pseudo-)optimality conditions for the value process $V_{k-1}$ and the hedge ratio $\delta_{k-1}$ :

$$
E\left(\Delta C_{k} \mid \mathcal{F}_{k-1}\right)=0
$$

and

$$
E\left(\left(\Delta C_{k}\right)\left(S_{k}-S_{k-1}+S_{k} \mathbf{g}^{\prime}\left(\delta_{k}-\delta_{k-1}\right)\right) \mid \mathcal{F}_{k-1}\right)=0 .
$$

Equation 2.11) can be better understood - especially when passing to the continuous time limit - by introducing a modified price process accounting for the cumulated effect of liquidity costs and market impact, as in 12$]$. To this end, we introduce the

Definition 2.3.1 The supply price $\bar{S}$ is the process defined by

$$
\bar{S}_{0}=S_{0}
$$

and, for $k \geqslant 1$,

$$
\bar{S}_{k}-\bar{S}_{k-1}=S_{k}\left(1+\mathbf{g}^{\prime}\left(\delta_{k}-\delta_{k-1}\right)\right)-S_{k-1},
$$

where $\mathbf{g}$ is defined in Equation 2.8.

Using $\bar{S}$ instead of $S$, Equation 2.11 can be rewritten as

$$
E\left(\left(\Delta C_{k}\right)\left(\bar{S}_{k}-\bar{S}_{k-1}\right) \mid \mathcal{F}_{k-1}\right)=0,
$$

again, a familiar expression in the context of local-risk minimization.

One can easily notice that Equations (2.10) and (2.11) reduce exactly to Equations (2.1) in [12] when market impact is neglected $(\gamma=0)$ and the risk function is quadratic.

Note that the approach in this section is similar in spirit, if somewhat easier from a technical point of view, to that in [8], where the bid-ask spread is taken into account and, therefore, the supply price becomes a non-smooth function of the increment $\delta_{k}-\delta_{k-1}$.

\section{The continuous-time setting}

This section is devoted to the characterization of the limiting equation for the value and the hedge parameter when the time step goes to zero. Since the proofs are identical to those given in [1] [12], we shall only provide formal derivations, limiting ourselves to the case of (continuous) Itō semi-martingales for the driving stochastic equations 


\subsection{The observed price dynamics}

A first result concerns the dynamics of the observed price. Assuming that the underlying processes are continuous and taking limits in ucp topology, one shows that the continuous-time equivalent of 2.2 is

$$
d S_{t}=S_{t}\left(d X_{t}+d A_{t}+\gamma \kappa^{\prime}(0) d \delta_{t}\right)
$$

where $X$ is a continuous martingale and $A$ is a continuous, predictable process of bounded variation. Equation (4.1) is fundamental in that it contains the information on the strategy-dependent volatility of the observed price that will lead to fully non-linear parabolic pricing equation. In fact, the following result holds true:

Lemma 3.1 Consider a hedging strategy $\delta$ which is a function of time and the observed price $S$ at time $t$ : $\delta_{t} \equiv \delta\left(S_{t}, t\right)$. Then, the observed price dynamics (4.1) can be rewritten as

$$
\left(1-\gamma \kappa^{\prime}(0) S_{t} \frac{\partial \delta}{\partial S}\right) \frac{d S_{t}}{S_{t}}=d X_{t}+d A_{t}^{\prime}
$$

where $A^{\prime}$ is another predictable, continuous process of bounded variation.

Proof: use Itō's lemma in Equation 4.1.

\subsection{Cost of a strategy and optimality conditions}

At this stage, we are not concerned with the actual optimality - with respect to local-risk minimization of pseudo-optimal solutions, but rather, with pseudo-optimality in continuous time. Hence, we shall use Equations 2.10 2.14 as a starting point when passing to the continuous time limit.

Thanks to $\mathbf{g}^{\prime}(0)=0$, there holds the

Proposition 3.2 The cost process of an admissible hedging strategy $(\delta, V)$ is given by

$$
C_{t} \equiv \int_{0}^{t}\left(d V_{u}-\delta d S_{u}+\frac{1}{2} S_{u} \mathbf{g}^{\prime \prime}(0) d<\delta, \delta>_{u}\right)
$$

Moreover, an admissible strategy is (pseudo-)optimal iff it satisfies the two conditions

- $C$ is a martingale

- $C$ is orthogonal to the supply price process $\bar{S}$, with

$$
d \bar{S}_{t}=d S_{t}+S_{t}\left(\mathbf{g}^{\prime \prime}(0) d \delta_{t}+\frac{1}{2} \mathbf{g}^{(3)}(0) d<\delta, \delta>_{t}\right) .
$$

In particular, if $C$ is pseudo-optimal, there holds that

$$
d<C, \bar{S}>_{t} \equiv d<V, S>_{t}-\delta d<S, S>_{t}+\mathbf{g}^{\prime \prime}(0) S_{t} d<V, \delta>_{t}-\delta S_{t} \mathbf{g}^{\prime \prime}(0) d<\delta, S>_{t}=0 .
$$

\section{Complete market: the single asset case}

It is of course interesting and useful to fully characterize the results of Section 3.2 in the case of a complete market. Hence, we assume in this section that the driving factor $X$ is a one-dimensional Wiener process $W$ and that $\mathcal{F}$ is its natural filtration, so that the increment of the observed price is simply

$$
d S_{t}=S_{t}\left(\sigma d W_{t}+\gamma \kappa^{\prime}(0) d \delta_{t}+d A_{t}\right)
$$


where the "unperturbed" volatility $\sigma$ is supposed to be constant. We also make the markovian assumption that the strategy is a function of the state variable $S$ and of time.

Under this set of assumptions, the orthogonality condition becomes trivial: the cost process $C$ has to be identically 0, Equation (3.5) yields

$$
\frac{\partial V}{\partial S}=\delta
$$

while the martingale condition for the cost process $C_{t}$ reads

$$
\frac{\partial V}{\partial t}+\frac{1}{2}\left(\frac{\partial^{2} V}{\partial S^{2}}+S_{t} \mathbf{g}^{\prime \prime}(0)\left(\frac{\partial^{2} V}{\partial S^{2}}\right)^{2}\right) \frac{d<S, S>_{t}}{d t}=0 .
$$

Applying Lemma 3.1 yields

$$
\left(1-\gamma \kappa^{\prime}(0) S_{t} \frac{\partial \delta}{\partial S}\right) \frac{d S_{t}}{S_{t}}=\sigma d W_{t}+d A_{t}^{\prime}
$$

leading to

$$
\frac{d<S, S>_{t}}{d t}=\frac{\sigma^{2} S_{t}^{2}}{\left(1-\gamma \kappa^{\prime}(0) S_{t} \frac{\partial \delta}{\partial S}\right)^{2}} .
$$

Hence, taking 4.2 into account, there holds

$$
\frac{\partial V}{\partial t}+\frac{1}{2}\left(\frac{\partial^{2} V}{\partial S^{2}}+\mathbf{g}^{\prime \prime}(0) S_{t}\left(\frac{\partial^{2} V}{\partial S^{2}}\right)^{2}\right) \frac{\sigma^{2} S_{t}^{2}}{\left(1-\gamma \kappa^{\prime}(0) S_{t} \frac{\partial \delta}{\partial S}\right)^{2}}=0
$$

or, using 4.2 and the identity $\mathbf{g}^{\prime \prime}(0)=(1-2 \gamma) \kappa^{\prime}(0)$ :

$$
\frac{\partial V}{\partial t}+\frac{1}{2}\left(\frac{\partial^{2} V}{\partial S^{2}}\left(1+(1-2 \gamma) \kappa^{\prime}(0) S_{t} \frac{\partial^{2} V}{\partial S^{2}}\right)\right) \frac{\sigma^{2} S_{t}^{2}}{\left(1-\gamma \kappa^{\prime}(0) S_{t} \frac{\partial^{2} V}{\partial S^{2}}\right)^{2}}=0
$$

Equation 4.7 can be seen as the pricing equation in our model: any contingent claim can be perfectly replicated at zero cost, as long as one can exhibit a solution to 4.7 . Consequently, of the utmost importance is the parabolicity of the pricing equation 4.7).

For instance, the case $\gamma=1$ corresponding to a full market impact (no relaxation) yields the following equation

$$
\frac{\partial V}{\partial t}+\frac{1}{2} \frac{\partial^{2} V}{\partial S^{2}} \frac{\sigma^{2} S_{t}^{2}}{\left(1-\gamma \kappa^{\prime}(0) S_{t} \frac{\partial^{2} V}{\partial S^{2}}\right)}=0
$$

which can be shown to be parabolic, see [11. In fact, there holds the sharp result

Theorem 4.1 Let us assume that $\frac{2}{3} \leqslant \gamma \leqslant 1$. Then, there holds:

- The non-linear backward partial differential operator

$$
V \rightarrow \frac{\partial V}{\partial t}+\frac{1}{2}\left(\left(1+(1-2 \gamma) \kappa^{\prime}(0) S_{t} \frac{\partial^{2} V}{\partial S^{2}}\right)\right) \frac{\sigma^{2} S_{t}^{2}}{\left(1-\gamma \kappa^{\prime}(0) S_{t} \frac{\partial^{2} V}{\partial S^{2}}\right)^{2}} \frac{\partial^{2} V}{\partial S^{2}}
$$

is parabolic.

- Every european-style contingent claim with payoff $\Phi$ satisfying the terminal constraint

$$
\sup _{S \in \mathbf{R}^{+}}\left(S \frac{\partial^{2} \Phi}{\partial S^{2}}\right)<\frac{1}{\gamma \kappa^{\prime}(0)}
$$

can be perfectly replicated via a $\delta$-hedging strategy given by the unique, smooth away from $T$, solution to Equation 4.7. 
Proof: the parabolic nature of the operator is determined by the monotonicity of the function

$$
p \rightarrow F(p)=\frac{p(1+(1-2 \gamma) p)}{(1-\gamma p)^{2}}
$$

A direct computation shows that $F^{\prime}(p)$ has the sign of $1+(2-3 \gamma) p$, so that $F$ is globally (in $p$ ) monotonic increasing on its domain of definition ] $-\infty, \frac{1}{\gamma}$ [ whenever $\frac{2}{3} \leqslant \gamma \leqslant 1$. Therefore, the pricing equation is globally well-posed in this parameter range.

\section{Complete market: the multi-asset case}

Consider a complete market described by $d$ state variables $X=X_{1}, \ldots, X_{d}$ : one can think for instance of a stochastic volatility model with $X_{1}=S$ and $X_{2}=\sigma$ when option-based hedging is available. 1

Using tradable market instruments, one is able to generate $d$ hedge ratio $\delta=\delta_{1}, \ldots, \delta_{d}$ with respect to the independent variables $X_{1}, \ldots, X_{d}$, that is, one can buy a combination of instruments whose price $P(t, X)$ satisfies

$$
\partial_{X_{i}} P=\delta_{i}
$$

We now introduce two market impact matrices, $\Lambda_{1}$ and $\Lambda_{2}$. The first one represents the virtual market impact and the second, the permanent impact. When $d=1$, they are linked to the previous notations by

$$
\Lambda_{1}=\kappa^{\prime}(0) S, \Lambda_{2}=\gamma \kappa^{\prime}(0) S
$$

Note that here, we proceed directly to the continuous time case, so that the actual shape of the order book plays a role only through its Taylor expansion around 0; hence, the use of the "linearized" impact via the matrices $\Lambda_{i}$.

The permanent market impact of a transaction $d \delta$ is given by

$$
d X=\Lambda_{2} \delta .
$$

The pricing equation is derived along the same lines as in Section 3 denote by $V$ the option price, and let $\Delta, \Gamma$ be the first and second derivatives of $V$. The relationship between the "unperturbed " price change $d \hat{X}_{t}$ and the observed price change $d X_{t}$ is given by

$$
d X_{t}=d \hat{X}_{t}+\Lambda_{2} d \delta_{t}+d A_{t}
$$

the $d$-dimensional version of 4.1. As before, a straightforward application of Itō's formula in a markovian setting yields the dynamics of the observed price

$$
d X=\left(I-\Lambda_{2} \Gamma\right)^{-1} d \hat{X}+d A_{t}^{\prime} .
$$

The $d$-dimensional version of 2.9 for the incremental cost of hedging is

$$
d C_{t}=d V_{t}-\sum_{i=1}^{d} \delta_{i} d X_{t}^{i}+\frac{1}{2} \operatorname{Trace}\left(\left(\Lambda_{1}-2 \Lambda_{2}\right) d<\delta, \delta>_{t}\right),
$$

so that, the market being complete, the perfect hedge condition $d C_{t}=0$ yields the usual delta-hedge

$$
\frac{\partial V}{\partial X_{i}}=\delta_{i}
$$

\footnotetext{
${ }^{1}$ In the next section, stochastic volatility is addressed in the context of an incomplete market
} 
together with the pricing equation

$$
\partial_{t} V+\frac{1}{2} \operatorname{Trace}\left(\Gamma \frac{d<X, X>_{t}}{d t}\right)=\operatorname{Trace}\left(\Gamma\left(\Lambda_{2}-\frac{1}{2} \Lambda_{1}\right) \frac{d<X, X>_{t}}{d t}\right) .
$$

Using (5.4), the pricing equation becomes

$$
\partial_{t} V+\frac{1}{2} \operatorname{Trace}\left[\left(\Gamma\left(I-\left(2 \Lambda_{2}-\Lambda_{1}\right) \Gamma\right)\right)\left(M \Sigma^{t r} M\right)\right]=0 .
$$

where we have set $\Sigma=\frac{d<\hat{X}, \hat{X}>_{t}}{d t}, M=\left(I-\Lambda_{2} \Gamma\right)^{-1}$.

When $\Lambda_{1}=\Lambda_{2}$ (i.e. no relaxation), the pricing equation becomes

$$
\partial_{t} V+\frac{1}{2} \operatorname{Trace}\left(\Gamma(I-\Lambda \Gamma)^{-1} \Sigma_{t}\right)=0 .
$$

which, degenerating further to the 1-dimensional case, yields the pricing equation already derived in 11

$$
\partial_{t} V+\frac{1}{2} \frac{\Gamma}{1-\lambda \Gamma} S^{2} \sigma^{2}=0 .
$$

The assessment of well-posedness in a general setting is related to the monotonicity of the linearized operator; in the case of full market impact $\Lambda_{1}=\Lambda_{2} \equiv \Lambda$, there holds the

Proposition 5.1 Assume that the matrix $\Lambda$ is symmetric. Then, Equation (5.10) is parabolic on the connected component of $\{\operatorname{det}(I-\Lambda \Gamma)>0\}$ that contains $\{\Gamma=0\}$.

Proof: let

$$
F(\Gamma)=\operatorname{Trace}\left(\Gamma(I-\Lambda \Gamma)^{-1} \Sigma_{t}\right)
$$

and

$$
H(\Gamma)=\Gamma(I-\Lambda \Gamma)^{-1}
$$

Denoting by $\mathbb{S}_{d}^{+}$the set of d-dimensional symmetric positive matrices, we need to show that for all $d \Gamma \in \mathbb{S}_{d}^{+}$, for all covariance matrix $\Sigma \in \mathbb{S}_{d}^{+}$, there holds

$$
F(\Gamma+d \Gamma) \geq F(\Gamma)
$$

Performing a first order expansion yields

$$
\begin{aligned}
H(\Gamma+d \Gamma)-H(\Gamma) & =\Gamma(I-\Lambda \Gamma)^{-1} \Lambda d \Gamma(I-\Lambda \Gamma)^{-1}+d \Gamma(I-\Lambda \Gamma)^{-1} \\
& =\left(\Gamma(I-\Lambda \Gamma)^{-1} \Lambda+I\right) d \Gamma(I-\Lambda \Gamma)^{-1}
\end{aligned}
$$

Using the elementary lemma 5.2 - stated below without proof - there immediately follows that

$$
\begin{aligned}
F(\Gamma+d \Gamma)-F(\Gamma) & =\operatorname{Trace}\left((I-\Gamma \Lambda)^{-1} d \Gamma(I-\Lambda \Gamma)^{-1} \Sigma\right) \\
& =\operatorname{Trace}\left(d \Gamma(I-\Lambda \Gamma)^{-1} \Sigma(I-\Gamma \Lambda)^{-1}\right) .
\end{aligned}
$$

Then, the symmetry condition on $\Lambda$ allows to conclude the proof of Proposition 5.1

Lemma 5.2 The following identity holds true for all matrices $\Gamma, \Lambda$ :

$$
\Gamma(I-\Lambda \Gamma)^{-1} \Lambda+I=(I-\Gamma \Lambda)^{-1} .
$$




\section{The case of an incomplete market}

In this section, stochastic volatility is considered. As said earlier, the results in Section 5 directly apply in this context whenever the market is assumed to be completed via an option-based hedging strategy. However, it is well known that such an assumption is equivalent to a very demanding hypothesis on the realization of the options dynamics and their associated risk premia, and it may be more realistic to assume that the market remains incomplete, and then, study a hedging strategy based on the tradable asset only. As we shall see below, such a strategy leads to more involved pricing and hedging equations.

Let then the observed price process be a solution to the following set of SDE's

$$
\begin{aligned}
d S_{t} & =S_{t}\left(\sigma_{t} d W_{t}^{1}+\gamma \kappa^{\prime}(0) d \delta_{t}+\mu_{t} d t\right) \\
d \sigma_{t} & =\nu_{t} d t+\Sigma_{t} d W_{t}^{2}
\end{aligned}
$$

where $\left(W^{1}, W^{2}\right)$ is a two-dimensional Wiener process under $\mathcal{P}$ with correlation $\rho$ :

$$
d<W^{1}, W^{2}>_{t}=\rho d t,
$$

and the processes $\mu_{t}, \nu_{t}$ and $\Sigma_{t}$ are actually functions of the state variables $S, \sigma$.

Consider again a markovian framework, thereby looking for the value process $V$ and the optimal strategy $\delta$ as smooth functions of the state variables

$$
\begin{aligned}
\delta_{t} & =\delta\left(S_{t}, \sigma_{t}, t\right) \\
V_{t} & =V\left(S_{t}, \sigma_{t}, t\right) .
\end{aligned}
$$

Then, the dynamics of the observed price becomes

$$
d S_{t}=\frac{S_{t}}{1-\gamma \kappa^{\prime}(0) S_{t} \frac{\partial \delta}{\partial S}}\left(\sigma_{t} d W_{t}^{1}+\gamma \kappa^{\prime}(0) \frac{\partial \delta}{\partial \sigma} d \sigma_{t}+d Q_{t}\right),
$$

the orthogonality condition reads

$$
\left(\frac{\partial V}{\partial S}-\delta\right) d<S, \bar{S}>_{t}+\frac{\partial V}{\partial \sigma} d<\sigma, \bar{S}>_{t}=0
$$

and the pricing equation for the value function $V$ is

$$
\begin{gathered}
\frac{\partial V}{\partial t}+\frac{1}{2}\left(\frac{\partial^{2} V}{\partial S^{2}}-\gamma \kappa^{\prime}(0) S_{t}\left(\frac{\partial \delta}{\partial S}\right)^{2}\right) \frac{d<S, S>_{t}}{d t}+\frac{1}{2}\left(\frac{\partial^{2} V}{\partial \sigma^{2}}-\gamma \kappa^{\prime}(0) S_{t}\left(\frac{\partial \delta}{\partial \sigma}\right)^{2}\right) \frac{d<\sigma, \sigma>_{t}}{d t}+ \\
+\left(\frac{\partial^{2} V}{\partial \sigma \partial S}-\gamma \kappa^{\prime}(0) S_{t} \frac{\partial \delta}{\partial \sigma} \frac{\partial \delta}{\partial S}\right) \frac{d<S, \sigma>_{t}}{d t}+\mathcal{L}_{1} V=0
\end{gathered}
$$

where $\mathcal{L}_{1}$ is a first-order partial differential operator.

Equations (6.4) and (6.5) are quite complicated. In the next paragraph, we focus on a particular case that allows one to fully assess their well-posedness.

\subsection{The case $\gamma=1, \rho=0$}

When $\gamma=1$, the martingale component of the supply price does not depend on the strategy anymore. As a matter of fact, the supply price dynamics is given by

$$
d \bar{S}_{t}=d S_{t}+S_{t}\left(\mathbf{g}^{\prime \prime}(0) d \delta_{t}+\frac{1}{2} \mathbf{g}^{(3)}(0) d<\delta, \delta>_{t}\right)
$$

and therefore, using

$$
\mathbf{g}^{\prime \prime}(0)=(1-2 \gamma) \kappa^{\prime}(0)=-\kappa^{\prime}(0),
$$


and the dynamics of $S$ in 6.1 , there holds that

$$
d \bar{S}_{t}=S_{t}\left(\sigma_{t} d W_{t}^{1}+d R_{t}\right)
$$

where $R$ is a predictable process of bounded variation.

If in addition, the Wiener processes for the asset and the volatility are supposed to be uncorrelated: $\rho=0$, the tedious computations leading to the optimal hedge and value function simplify, and one can study in full generality the well-posedness of the pricing and hedging equations $(6.4)(6.5)$.

First and foremost, the orthogonality condition 6.4 simply reads in this case

$$
\delta=\frac{\partial V}{\partial S}
$$

exactly as in the complete market case. This is a standard result in local-risk minimization with stochastic volatility when there is no correlation.

As for the pricing equation (6.5), one first works out using (6.8) the various brackets in 6.5 and finds that

$$
\begin{gathered}
\frac{d<S, S>_{t}}{d t}=\left(1-\kappa^{\prime}(0) S_{t} \frac{\partial^{2} V}{\partial S^{2}}\right)^{-2}\left(\sigma_{t}^{2} S_{t}^{2}+\kappa^{\prime}(0)^{2} S_{t}^{2}\left(\frac{\partial^{2} V}{\partial S \partial \sigma}\right)^{2} \Sigma_{t}^{2}\right), \\
\frac{d<\sigma, \sigma>_{t}}{d t}=\Sigma^{2}
\end{gathered}
$$

and

$$
\frac{d<S, \sigma>_{t}}{d t}=\left(1-\kappa^{\prime}(0) S_{t} \frac{\partial^{2} V}{\partial S^{2}}\right)^{-1} \kappa^{\prime}(0) S_{t} \Sigma_{t}^{2} \frac{\partial^{2} V}{\partial S \partial \sigma} .
$$

Plugging these expressions in 6.5 yields the pricing equation for $V$

$$
\begin{gathered}
\frac{\partial V}{\partial t}+\frac{1}{2} \frac{\partial^{2} V}{\partial S^{2}}\left(1-\kappa^{\prime}(0) S_{t}\left(\frac{\partial^{2} V}{\partial S^{2}}\right)\right)^{-1}\left(\sigma_{t}^{2} S_{t}^{2}+\kappa^{\prime}(0)^{2} S_{t}^{2}\left(\frac{\partial^{2} V}{\partial S \partial \sigma}\right)^{2} \Sigma_{t}^{2}\right)+\frac{1}{2}\left(\frac{\partial^{2} V}{\partial \sigma^{2}}-\kappa^{\prime}(0) S_{t}\left(\frac{\partial^{2} V}{\partial S \partial \sigma}\right)^{2}\right) \Sigma^{2}+ \\
\kappa^{\prime}(0) S_{t} \Sigma_{t}^{2}\left(\frac{\partial^{2} V}{\partial S \partial \sigma}\right)^{2}+\mathcal{L}_{1} V=0
\end{gathered}
$$

or, after a few final rearrangments,

$$
\frac{\partial V}{\partial t}+\frac{\sigma_{t}^{2} S_{t}^{2}}{2\left(1-\kappa^{\prime}(0) S_{t}\left(\frac{\partial^{2} V}{\partial S^{2}}\right)\right)} \frac{\partial^{2} V}{\partial S^{2}}+\frac{1}{2} \frac{\partial^{2} V}{\partial \sigma^{2}} \Sigma^{2}+\frac{1}{2} \frac{\kappa^{\prime}(0) S_{t} \Sigma^{2}}{\left(1-\kappa^{\prime}(0) S_{t}\left(\frac{\partial^{2} V}{\partial S^{2}}\right)\right)}\left(\frac{\partial^{2} V}{\partial \sigma \partial S}\right)^{2}+\mathcal{L}_{1} V=0 .
$$

The main result of this section is the

Proposition 6.1 Equation 6.13 is of parabolic type.

Proof: one has to study the monotocity of the operator

$$
\mathcal{L}: V \rightarrow \mathcal{L}(V) \equiv \frac{\sigma_{t}^{2} S_{t}^{2}}{2\left(1-\kappa^{\prime}(0) S_{t}\left(\frac{\partial^{2} V}{\partial S^{2}}\right)\right)} \frac{\partial^{2} V}{\partial S^{2}}+\frac{1}{2} \frac{\partial^{2} V}{\partial \sigma^{2}} \Sigma^{2}+\frac{1}{2} \frac{\kappa^{\prime}(0) S_{t} \Sigma^{2}}{\left(1-\kappa^{\prime}(0) S_{t}\left(\frac{\partial^{2} V}{\partial S^{2}}\right)\right)}\left(\frac{\partial^{2} V}{\partial \sigma \partial S}\right)^{2}
$$

Introducing the classical notations

$$
p \equiv\left(\begin{array}{ll}
p_{11} & p_{12} \\
p_{21} & p_{22}
\end{array}\right)
$$

with $p_{11}=\frac{\partial^{2} V}{\partial S^{2}}, p_{12}=p_{21}=\frac{\partial^{2} V}{\partial S \partial \sigma}$ and $p_{22}=\frac{\partial^{2} V}{\partial \sigma^{2}}$ and defining

$$
\mathbf{L}(S, \mathbf{p}) \equiv \frac{\sigma_{t}^{2} S_{t}^{2} p_{11}}{\left(1-\kappa^{\prime}(0) S_{t} p_{11}\right)}+\Sigma^{2} p_{22}+\frac{\kappa^{\prime}(0) S_{t} \Sigma^{2}}{\left(1-\kappa^{\prime}(0) S_{t} p_{11}\right)} p_{12}^{2}
$$


one is led to study the positivity of the $2 \times 2$ matrix

$$
\left(\begin{array}{cc}
\frac{\partial \mathbf{L}}{\partial p_{11}} & \frac{1}{2} \frac{\partial \mathbf{L}}{\partial p_{12}} \\
\frac{1}{2} \frac{\partial \mathbf{L}}{\partial p_{12}} & \frac{\partial \mathbf{L}}{\partial p_{22}}
\end{array}\right) .
$$

Setting $F\left(p_{11}\right)=\frac{\sigma^{2} S^{2} p_{11}}{1-\kappa^{\prime}(0) S p_{11}}$ and $D\left(p_{11}\right)=1-\kappa^{\prime}(0) S p_{11}$, one needs to show that the matrix $\mathbf{H}(\mathbf{p})$

$$
\left(\begin{array}{cc}
F^{\prime}\left(p_{11}\right)+\left(\kappa^{\prime}(0) S \Sigma\right)^{2} \frac{p_{12}^{2}}{D^{2}} & \kappa^{\prime}(0) S \Sigma^{2} \frac{p_{12}}{D} \\
\kappa^{\prime}(0) S \Sigma^{2} \frac{p_{12}}{D} & \Sigma^{2}
\end{array}\right)
$$

is positive. This result is trivially shown to be true by computing the trace and determinant of $\mathbf{H}(\mathbf{p})$ :

$$
\operatorname{Tr}(\mathbf{H}(\mathbf{p}))=F^{\prime}\left(p_{11}\right)+\Sigma^{2}+\left(\kappa^{\prime}(0) S \Sigma\right)^{2} \frac{p_{12}^{2}}{D^{2}}
$$

and

$$
\operatorname{Det}(\mathbf{H}(\mathbf{p}))=\Sigma^{2} F^{\prime}\left(p_{11}\right)
$$

and using the fact that $F$ is a monotonically increasing function.

This ends the proof of Proposition 6.1.

As a final remark, we point out that the condition on the payoff for 6.13 to have a global, smooth solution, is exactly the same as in the one-dimensional case: stochastic volatility does not impose further constraints, except the now imperfect replication strategy.

\section{Conclusion}

In this work, we model the effect of liquidity costs and market impact on the pricing and hedging of derivatives, using a static order book description and introducing a numerical parameter measuring the level of asymptotic market impact. In the complete market case, a structural result characterizing the well-posedness of the associated, strategy-dependent diffusion, is proven. Extensions to incomplete markets and nonlinear hedging strategies are also considered.

Of interest is the interpretation of the condition on the resilience parameter: $\frac{2}{3} \leqslant \gamma \leqslant 1$. The case $\gamma>1$ is rather trivial to understand, as one can easily see that it leads to arbitrage by a simple round-trip trade. The case $\gamma<\frac{2}{3}$ is not so simple. The loss of monotonicity of the function $F(p)=\frac{p(1+(1-2 \gamma) p)}{(1-\gamma p)^{2}}$ for $\gamma<\frac{2}{3}$ yields the existence of $p_{1}, p_{2}$ such that $p_{1}<p_{2}$ but $F\left(p_{1}\right)>F\left(p_{2}\right)$. Hence, one can buy at a cheaper price an option with higher convexity at maturity. However, it is worth noting that, due to the nonlinearity of the pricing equation, one cannot simply build an arbitrage portfolio by holding a long/short position in two options, so that it is not necessarily possible to transform this reverse inequality into an arbitrage opportunity.

Another important question has been left aside so far: the behaviour of the solution to the pricing equation when the constraint is violated at maturity - after all, this is bound to be the case for a real-life contingent claim such as a call option! From a mathematical point of view, see the discussion in [11, there is a solution which amounts to replace the pricing equation by a variational inequality, but of course, in this case, the perfect replication does not exist any longer. Rather, one may want to use, in a vein similar to [4] where pricing with constraints on the " $\Gamma$ " of the option is studied, a super-replicating strategy.

\section{References}

[1] F. Abergel and N. Millot. Non quadratic local risk-minimization for hedging contingent claims in incomplete markets. SIAM Journal on Financial Mathematics, 2(1):342-356, 2011. 
[2] R. Almgren, C. Thum, E. Hauptmann, and H. Li. Direct estimation of equity market impact. working paper.

[3] U. Cetin, R. Jarrow, and P. Protter. Liquidity risk and arbitrage pricing theory. Finance and Stochastics, 8:311-341, 2004.

[4] U. Çetin, H. M. Soner, and N. Touzi. Option hedging for small investors under liquidity costs. Finance Stoch., 14(3):317-341, 2010.

[5] R. Frey. Perfect option hedging for a large trader. 1996.

[6] R. Frey and A. Stremme. Market volatility and feedback effects from dynamic hedging. Mathematival Finance, 7(4):351-374, 1997.

[7] P. Henry-Labordère. working paper.

[8] D. Lamberton, H. Pham, and M. Schweizer. Local risk-minimization under transaction costs. Mathematics of Operations Research, 23:585-612, 1997.

[9] H. E. Leland. Option pricing and replication with transactions costs. Journal of Finance, 40(5):1283$1301,1985$.

[10] H. Liu and J. M. Yong. Option pricing with an illiquid underlying asset market. Journal of Economic Dynamics and Control, 29:2125-2156, 2005.

[11] G. Loeper. Option pricing with market impact and non-linear black and scholes pde's. http://arxiv.org/abs/1301.6252, 2013.

[12] N. Millot and F. Abergel. Non quadratic local risk-minimization for hedging contingent claims in the presence of transaction costs. Available at SSRN 1881175, 2011.

[13] Eckhard Platen and Martin Schweizer. On feedback effects from hedging derivatives. Mathematical Finance, 8(1):67-84, 1998.

[14] A. Roch. Liquidity risk, volatility and financial bubbles. PhD Thesis.

[15] J. Schönbucher, P. and P. Wilmott. The feedback effect of hedging in illiquid markets. SIAM J. Appl. Maths, 61(1):232-272, 2000.

[16] P. Weber and B. Rosenow. Order book approach to price impact. Quantitative Finance, 5(4):357-364, 2005.

[17] V. Zakamouline. European option pricing and hedging with both fixed and proportional transaction costs. working paper. 